\title{
WATER WAKE
}

\section{KRISTEN ROBERTS}

They drive in procession to Point Addis

(a place where land ends abruptly above the sea

and the waves corrupt its flaws incessantly)

and walk in silence down to the beach.

The wreath stays on the board a while

(unsettling with each undulation

as the sea-floor drops and swell increases)

and then dark water seeping sets it free.

Most of them pocket a stone or a shell (and one remarks that flowers make no sound in the wind; that if cliffs whistle and wave tips hiss, the silence of flowers feels grim) and as they climb the stairs to the road they sing, for songs were his other great love. 